\title{
Analysis of Interference Signals Generated by Vibroacoustic Vibrations of Transformer Cores
}

\author{
T. BOCZAR*, S. Borucki AND A. Cichoń \\ Institute of Power Engineering and Renewable Energy, Faculty of Electrical Engineering, Automatic Control \\ and Computer Science, Opole University of Technology, Prószkowska 76, 45-758 Opole, Poland
}

\begin{abstract}
The article deals with the subject matter referring to the interference that may accompany measurements of vibroacoustic signals, generated by electric power transformers, taken by using the acoustic emission method under industrial conditions. Paper concerns measurement and analysis of internal, acoustic type disturbances, including the magnetostrictive vibrations of plate sets within the transformer core, caused by changes in the magnetic flux. Paper presents measurement results of acoustic interfering signals and results of analyses made in the frequency and time-frequency domains. Power spectral density, MUSIC pseudospectra, two- and three-dimensional short time Fourier transform spectrograms, continuous wavelet transform scalograms, discrete wavelet transform, and quantities of energy transferred are included in the paper.
\end{abstract}

DOI: 10.12693/APhysPolA.125.1355

PACS: $79.60 . \mathrm{Bm}, 42.79 . \mathrm{Pw}, 52.80 . \mathrm{Hc}$

\section{Introduction}

During diagnostics of paper-oil insulation systems of electric power equipment by using the acoustic method, one needs to consider the possibility of interfering signals, which may affect the registered acoustic emission (AE) signals. The presence of interfering signals during the measurements may impact the correctness of evaluation of the obtained results and in consequence prevent effective partial discharges (PD) form identification, location detection of their standard occurrence, and above all proper recognition of defects in the tested insulation.

Distortions, which may occur during diagnostic measurements performed using the AE method under industrial conditions, can be divided into internal that are present in the tank of the test transformer and external, which occur outside the diagnosed object. They are related to the technical and environmental conditions of the place of installation. Internal and external distortions can be divided with respect to the source of their generation into acoustic, electrical and mechanical distortions.

Internal distortions of acoustic and electric nature may not only affect the propagation conditions of $\mathrm{AE}$ waves from PD generated in the insulating system of the test object but also may affect particular elements of the measurement chain. External acoustic and electric interferences affect the measuring system as well as the tested transformer. Instead external mechanical distortions may only have an impact on measurement chain used during diagnostic.

This article concerns measurement and analysis of internal disturbances of acoustic type, which are magne-

*corresponding author; e-mail: tboczar@gmail.com tostrictive vibrations of plate sets in transformer core, caused by changes in the magnetic flux. The magnetostriction phenomenon is an elastic deformation of magnetic materials during their magnetization caused by changes in the orientation of ferromagnetic domains. This phenomenon is the primary cause of vibrations in metal plates forming the core of the transformer, which in turn are sources of the generated interfering acoustic signals. Transformer magnetic circuit undergoes cyclical changes in its magnetic direction, and the frequency of the resulting vibrations is more than twice greater than in the network. They may also contain higher harmonics, caused by the phenomenon of magnetic hysteresis. Magnetostriction intensity can be reduced by: selection of appropriate mechanical and thermal processes of metal plates forming a transformer core; adjustment of magnetic flux value in the core of the transformer; appropriate core structure that is inter alia appropriate fitting (compression) of individual plate sets [1].

Transforms with rating power not exceeding tens of $\mathrm{kVA}$ produce interfering signals with sound pressure at the level of $40 \mathrm{~dB}(\mathrm{~A})$. High power units (several hundred of MVA) may produce interfering signals at the level of up to $90 \mathrm{~dB}(\mathrm{~A})$. Magnetostrictive interfering signal spectrum may cover ranges up to $40 \mathrm{kHz}$, which depends largely on the structure and rating power of the transformer [1]. The authors in [2] conducted comparative research of various design solutions of high power transformers and have defined a representative magnetostrictive interfering signal bandwidth in the range from $10 \mathrm{kHz}$ to $65 \mathrm{kHz}$. However, in [3] it was stated that higher harmonics, occurring as a result of magnetic hysteresis loop, can cause situation, in which the bandwidth of interfering signals coming from magnetostriction phenomenon will be in the range $(30-60) \mathrm{kHz}$. Even a wider range of these interfering signals, covering the range of up to $80 \mathrm{kHz}$, was indicated on the basis of the research 
made by authors of [4]. Issues related to reduction of interfering acoustic signal caused by operation of power transformers were described in detail, among others in $[1,5-18]$.

\section{Characteristics of the circuit modeling interfering acoustic signals generated by magnetostrictive vibrations of transformer core}

To model the phenomenon of magnetostrictive vibrations of metal plate package forming the transformer core, a brand new three-phase transformer with a solid insulation was used. Figure 1 shows technical drawing, which includes its geometrical dimensions, while in Table its basic nominal data was summarized.
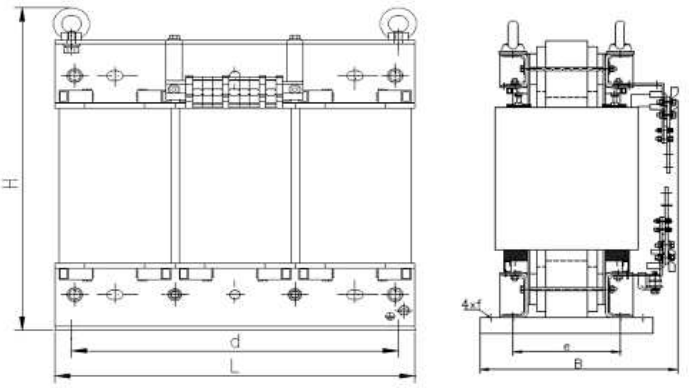

Fig. 1. Technical drawing of the test transformer, where $H=545 \mathrm{~mm}, L=540 \mathrm{~mm}, d=490 \mathrm{~mm}$, $B=320 \mathrm{~mm}, e=220 \mathrm{~mm}, f=13 \times 18 \mathrm{~mm}^{2}$ [19].

TABLE

Selected nominal parameters of the test transformer.

\begin{tabular}{l|c}
\hline \multicolumn{1}{c|}{ Nominal parameter } & Value \\
\hline power & $40 \mathrm{kVA}$ \\
primary voltage & $600 \mathrm{~V}$ \\
secondary voltage & $30 \mathrm{~V}$ \\
primary winding current & $17.9 \mathrm{~A}$ \\
secondary winding current & $354 \mathrm{~A}$ \\
no-load losses & $255 \mathrm{~W}$ \\
short losses & $286 \mathrm{~W}$ \\
percentage of short-circuit voltage & $3.67 \%$
\end{tabular}

\section{Methodology of measurements performed in the study}

During the measurements, the test transformer was loaded with a water resistor (Fig. 2). The load current, measured on the $600 \mathrm{~V}$ side, was equal to $354 \mathrm{~A}$. The range of performed registrations of interfering acoustic signals generated by the magnetostriction phenomenon in the transformer core, covered the following cases:

- assessment of effects on the obtained results of transducer position changes placed on the surface of the core yoke. For this purpose, measurements were performed while changing the position of the transducer along the length of the yoke (labelled with the letter $\mathrm{L}$ in Fig. 1), by performing registrations with gradations of $1 \mathrm{~cm}$. The research tests were performed on both: the lower and the upper yoke of the transformer magnetic circuit, at the clamping force constituting $5 \%$ of the factory torsion;

- assessment of the influence between the clamping force acting on plate packets forming the transformer core on the obtained measurement results. For this purpose, interfering acoustic signals were recorded for five cases:

- factory clamped core (100\% clamping force),

- reduction of the clamping force, which corresponds to: $80 \%, 60 \%, 50 \%, 25 \%$ and $10 \%$ (unclamped core) of the force value applied during production of the clamping plates. In each of the researched cases, the transducer was in the same place. However, the all six screws, which press the examined transformer core were loosened equally.

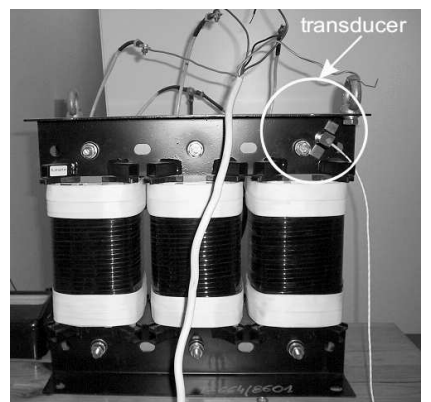

Fig. 2. View of the examined transformer with measurement transducer installed on the core yoke.

For measurements of the acoustic signal interferences a standard system from Physical Acoustics Corporation (PAC) was used. The measurement chain consists of the following elements: wideband measurement transducer WD, type $\mathrm{AH} 17$, preamplifier type $2 / 4 / 6$ from PAC, broadband amplifier of type AE2A, a 12-bit measurement card type CH-3160, and industry PC. Detailed description of the measurement chain and the procedures used for processing and analyzing the recorded signals are presented in $[1,7,10]$.

\section{Frequency and time-frequency analysis of the obtained results}

On the basis of statistical tests it was determined, allowing $5 \%$ to error, that the position change of the transducer within the tested yokes does not have statistically significant effect on the obtained measurement results. This article presents the results for the pre-turned core, 
which pose a reference for the results obtained with the loosened core (clamping force represented $10 \%$ of the force value for the pre-turned core case). For the presented results the test transducer was placed on the upper yoke (at a distance of $3 \mathrm{~cm}$ from the right edge of the core), as shown in Fig. 2. For changes of the clamping force statistically different results were obtained, wherein the impact of screwing size was most notable for the weakest clamping ( $10 \%$ of the pre-turned force).
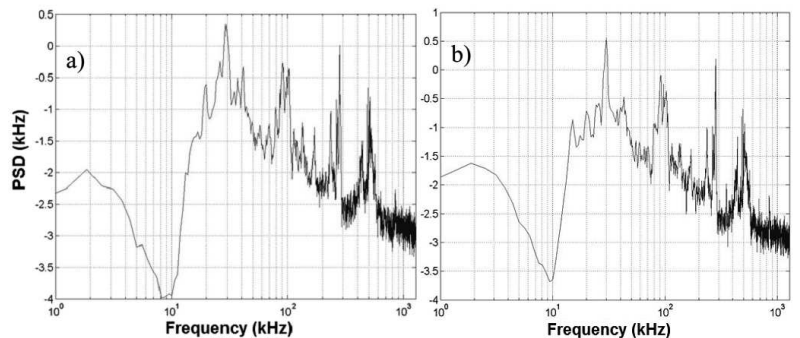

Fig. 3. PSD of interfering acoustic signal generated by magnetostrictive vibrations of transformer core, where: (a) turned core and (b) loosened core.

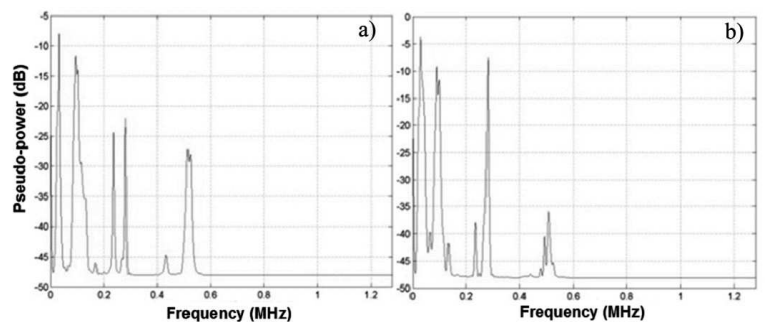

Fig. 4. MUSIC pseudospectrum of interfering acoustic signals generated by magnetostrictive vibrations of transformer core, where: (a) turned core and (b) loosened core.

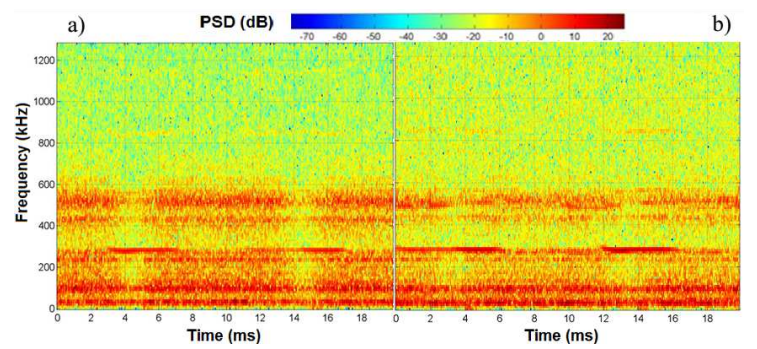

Fig. 5. STFT spectrogram of core signals generated by magnetostrictive vibrations of transformer, where: (a) turned core and (b) loosened core.

Figures 3 to 10 present the following characteristics: power density spectra (Fig. $3 \mathrm{a}$ and b), MUSIC pseudospectra (Fig. 4a and b), two-dimensional short time Fourier transform (STFT) spectrograms (Fig. 5a and b), three-dimensional amplitude spectrograms (Fig. 6a and b), power density (Fig. 7a and b), continuous wavelet transform (CWT) scalograms (Fig. 8a,b),

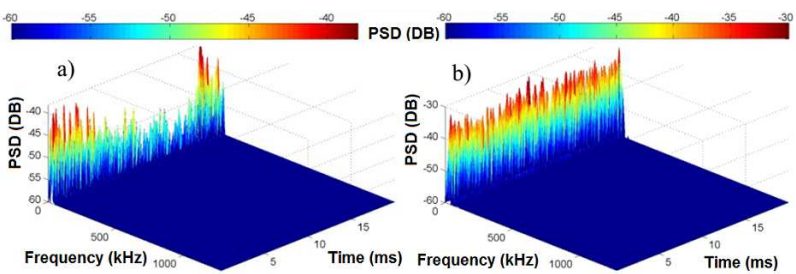

Fig. 6. PSD time-frequency spectrogram of interfering acoustic signals generated by magnetostrictive vibrations of transformer core, where: (a) turned core and (b) loosened core.

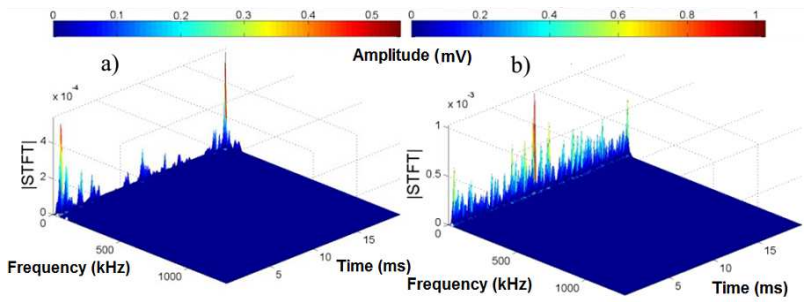

Fig. 7. Amplitude spectrogram of interfering signals generated by magnetostrictive vibrations of transformer core, where: (a) turned core and (b) loosened core.

waveforms of wavelet decomposition (DWT) (Fig. 9a,b) and energy quantity transferred by the particular details (Fig. 10a and b).

\section{Summary}

By analyzing the results derived from frequency analysis, obtained using fast Fourier transform and MUSIC pseudospectral transformation for the examined acoustic interfering signals caused by the magnetostriction phenomenon, it can be concluded that:

a) for the pre-turned core, on the obtained waveforms three characteristic ranges can be distinguished:

- 6-320 kHz the largest energy participation in the analyzed spectrum and pseudospectrum, wherein in this band seven peaks of narrowband extremes can be observed in the following frequency ranges: $6-30 \mathrm{kHz}, 60-105 \mathrm{kHz}$,

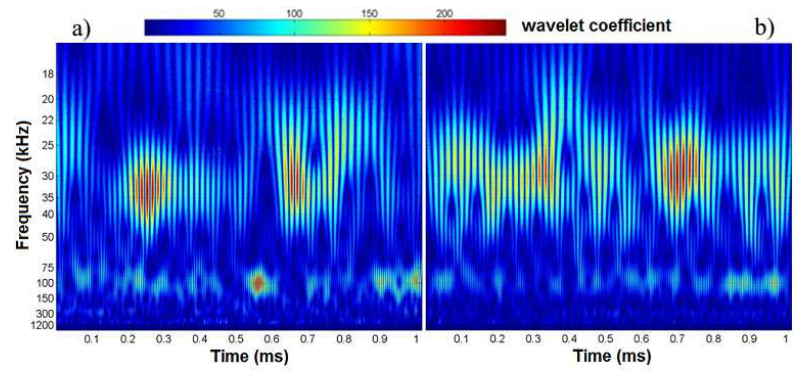

Fig. 8. CWT of interfering signals generated by magnetostrictive vibrations of transformer core, where: (a) turned core and (b) loosened core. 


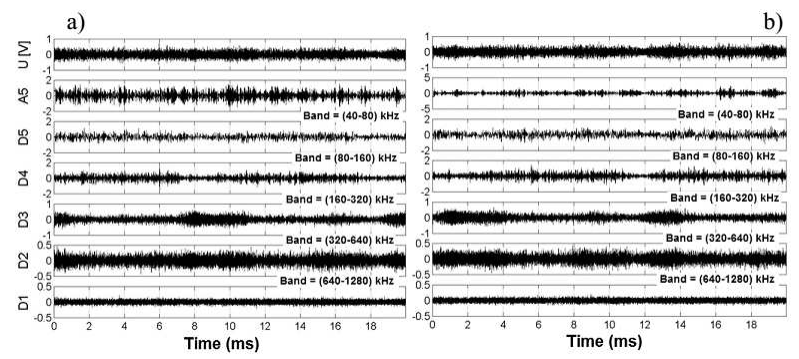

Fig. 9. DWT by particular interfering signal details generated by magnetostrictive vibrations of transformer core, where: (a) turned core and (b) loosened core.

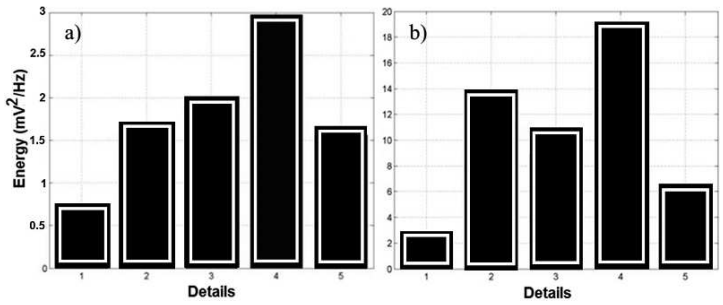

Fig. 10. Quantity of energy transferred by particular interfering signal details generated by magnetostrictive vibrations of transformer core, where: (a) turned core and (b) loosened core.

$115-145 \mathrm{kHz}, 150-175 \mathrm{kHz}, 205-215 \mathrm{kHz}, 225-$ $250 \mathrm{kHz}$, and $265-295 \mathrm{kHz}$,

- $300-630 \mathrm{kHz}$ the amplitude is on average lower of about $15 \mathrm{~dB}$ as compared to the above-mentioned range; there are two dominant extremes in the bands: $410-435 \mathrm{kHz}$ and 500 $570 \mathrm{kHz}$,

- above $635 \mathrm{kHz}$ a flat spectrum with the lowest power values, a decrease of about $7 \mathrm{~dB}$ in comparison to the previous case and about $17 \mathrm{~dB}$ relative to the first case.

b) for the loosened core, as in the case of the pre-turned one, on the determined waveforms of PSD and MUSIC pseudospectra three characteristic frequency ranges can be distinguished:

- $15-320 \mathrm{kHz}$, which is dominant in the entire waveform in terms of energy transfer amount. In this range, as in the previous case, seven local narrowband and peak-type extrema are visible that are present in the following ranges: $0-45 \mathrm{kHz}, 55-70 \mathrm{kHz}, 85-$ $110 \mathrm{kHz}, 120-140 \mathrm{kHz}, 145-160 \mathrm{kHz}, 235-$ $245 \mathrm{kHz}$, and $285-310 \mathrm{kHz}$,

- 380-590 kHz, in this band the amplitude, which is in average $9 \mathrm{~dB}$ lower than in the first case, there are two dominant broadband extrema in the ranges: $410-460 \mathrm{kHz}$ and $495-$ $580 \mathrm{kHz}$,
- 310-385 kHz and above $580 \mathrm{kHz}$, a flat spectrum with the smallest amplitude values, at average $23 \mathrm{~dB}$ lower, as compared to the first dominant frequency band.

On the spectrograms determined for the interfering acoustic signals generated by the magnetostrictive vibrations for both: the pre-turned as well as for the loosened bolts cores, dominant bands in the range from 0 to $600 \mathrm{kHz}$ are visible, which have the maximum power (amplitude) in the frequency band from 0 to $90 \mathrm{kHz}$ through the entire analyzed waveform.

By analyzing the time-frequency structures illustrated in scalograms calculated by using the CWT, the following can be concluded:

a) for the pre-turned core plates, wavelet images are visible in two frequency ranges: $20-50 \mathrm{kHz}$ and 80 $135 \mathrm{kHz}$. Wavelet images occurring in the first band with an average duration of approximately $0.14 \mathrm{~ms}$, have fringe structure (from 8 to 12 fringes in a single image). They have lower amplitude and intensity as compared to the coherent structures generated in the second frequency band, the average duration of which is in the range from 0.03 to $0.1 \mathrm{~ms}$.

b) for the loosened core plates, wavelet images are at the same frequency bands as in the previous case, i.e.: $25-55 \mathrm{kHz}$ and $75-135 \mathrm{kHz}$.

On the basis of the multiresolution analysis results obtained using DWT it can be concluded that for both cases the highest energy is transferred through the fourth detail (D4), while the lowest one through the first detail (D1). The amount of energy for the second, third and fifth levels of decomposition is comparable and is at the level of approximately $50 \%$ of the energy carried by the $\mathrm{D} 4$ detail. On the waveforms, there are visible active structures with duration of approximately $1.5 \mathrm{~ms}$; in particular for the D4 and D3 details for the pre-turned core. While, for the loosened core, there are visible impulse series on second and fourth level of decomposition with longer duration, which constitutes about $3.5 \mathrm{~ms}$.

\section{Acknowledgments}

The work was financed by the National Research and Development Center within the LIDER program.

\section{References}

[1] T. Boczar, S. Borucki, D. Zmarzły, in: Proc. 39th Int. Congr. Exp. Noise Contr. Eng. InterNoise, Lisbon 2010, paper 301.

[2] Z.T. Deheng Kexiong, T.J. Xianche, in: II Conf. Prop. Appl. Diel. Mater., Vol. 2, Bejing 1988, p. 614.

[3] E. Howells, E.T. Norton, IEEE-PAS-100 1, 158 (1981).

[4] A.M.L.T. Bianchi, S. Mainardi, Cerutti, Trans. Inst. Measur. Control 3, 215 (2002). 
[5] S.M. Markalous, S. Tenbohlen, K. Feser, IEEE Trans. DEI 15, 1576 (2008).

[6] J. Fuhr, IEEE Trans. DEI 12, 1005 (2005).

[7] T. Boczar, S. Borucki, A. Cichoń, D. Zmarzły, IEEE Trans. DEI 16, 214 (2009).

[8] H. Zhang, T.R. Blackburn, B.T. Phung, D. Sen, IEEE Trans. DEI 14, 15 (2007).

[9] M.S. Naderi, M. Vakilian, T.R. Blackburn, B.T. Phung, A. Nasiri, IEEE Trans. DEI 14, 436 (2007).

[10] D. Wotzka, D. Zmarzły, T. Boczar, Acta Phys. Pol. A 118, 1272 (2010).

[11] L. Tang, R. Luo, M. Deng, J. Su, IEEE Trans. DEI 15, 492 (2008).

[12] P. Kundu, N.K. Kishore, A.K. Sinha, Appl. Acoust. 70, 1378 (2009).

[13] D. Wotzka, T. Boczar, P. Frącz, Acta Phys. Pol. A 120, 767 (2011).
[14] L. Junhao, H. Quanwei, Z. Xuefeng, Y. Xiu, Y. Yongfen, Y. Yanming, IEEE Trans. Power Deliv. 26, 538 (2011).

[15] L. Fuqi, J. Lei, Adv. Technol. Electr. Eng. 19, 69 (2000).

[16] D. Aschenbrenner, H.G. Kranz, W.R. Rutgers, P. Van den Aardweg, IEEE Trans. DEI 12, 216 (2005).

[17] S.M. Strachan, S. Rudd, S.D.J. McArthur, M.D. Judd, S. Meijer, E. Gulski, IEEE Trans. DEI 1, 259 (2008).

[18] T. Boczar, D. Zmarzły, IEEE Trans. DEI 13, 632 (2006).

[19] www.elhand.pl. 